\title{
Optimization of piezoelectric bimorph actuators with active damping for static and
} dynamic loads

\section{Donoso, Alberto; Sigmund, Ole}

\section{Published in:}

Structural and Multidisciplinary Optimization

Link to article, DOI:

$10.1007 / \mathrm{s} 00158-008-0273-0$

Publication date:

2009

Document Version

Early version, also known as pre-print

Link back to DTU Orbit

\section{Citation (APA):}

Donoso, A., \& Sigmund, O. (2009). Optimization of piezoelectric bimorph actuators with active damping for static and dynamic loads. Structural and Multidisciplinary Optimization, 38(2), 171-183. https://doi.org/10.1007/s00158008-0273-0

\section{General rights}

Copyright and moral rights for the publications made accessible in the public portal are retained by the authors and/or other copyright owners and it is a condition of accessing publications that users recognise and abide by the legal requirements associated with these rights.

- Users may download and print one copy of any publication from the public portal for the purpose of private study or research.

- You may not further distribute the material or use it for any profit-making activity or commercial gain

- You may freely distribute the URL identifying the publication in the public portal 


\title{
Optimization of piezoelectric bimorph actuators with active damping for static and dynamic loads
}

\author{
Received: date / Revised: date
}

\begin{abstract}
The paper considers optimal design problems in the context of active damping. More specifically, we are interested in controlling the tip-deflection of a cantilever beam subjected to static and time-harmonic loading on its free extreme. First, the thickness profile of a piezoelectric bimorph actuator is optimized and second, the width profile. In the thickness study, formulation and results depend on whether the electric field or the applied voltage is kept constant. For the latter case we propose a differentiable model that connects electric field and piezo-actuator thickness to include electric field breakdown. Results are presented for both design variable cases, for static as well as for dynamic excitation for single frequency and frequency intervals.
\end{abstract}

Keywords Variable thickness - Variable width · Piezoelectricity · Bimorph actuators - Shape optimization

\section{Introduction}

Since the piezoelectric effect was first discovered in 1880 by the French scientists Pierre and Paul-Jacques Curie, the use of piezoelectric materials has really increased in modern engineering applications, especially in the field of smart structures, where they are used as actuators and/or sensors. As actuators they have the property of

The major part of this research was performed while A. Donoso was a visiting researcher at the Department of Mechanical Engineering, Technical University of Denmark.
A. Donoso
Departamento de Matemáticas, ETS de Ingenieros Industri- ales, Universidad de Castilla La Mancha, 13071 Ciudad Real (Spain)
Tel.: +34-926-295300 (3859)
Fax: +34-926-295361
E-mail: Alberto.Donoso@uclm.es

\section{O. Sigmund}
Department of Mechanical Engineering, Section of Solid Me- chanics, Technical University of Denmark, Nils Koppels Alle, Building 404, DK-2800, Kgs. Lyngby (Denmark).
E-mail: sigmund@mek.dtu.dk

converting electrical energy into mechanical, that is, they strain under an applied voltage. As sensors they do the opposite, they produce an electric signal when deformed.

Although the magnitudes of piezoelectric displacements and/or voltages are small, piezoelectric materials have been adapted to an impressive range of applications. An interesting application is the active control of structural vibrations. As opposed to passive damping, which often adds unwanted weight to the structure, active damping can reduce vibrations with little increase in weight. Active damping consists in adding piezoelectric actuators and sensors to the structure to be controlled. These actuators and sensors are usually patches embedded in laminated composites or surface bonded to flexible structures such as beams or plates, forming the so-called smart structures. In practice, the most common piezoelectric materials used in this field are twofold: PZT (lead zirconate titanate) [10] and PVDF (polyvinylidene fluoride) [11]. They are basically chosen due to their light weight, relatively low cost, small size and good frequency response. More specifically, the brittleness and stiffness of PZT makes it well suited to be used as actuator, while the compliance of PVDF makes it a better candidate for sensing applications.

It is obvious that the efficiency of actively controlled systems depends very much on the number, position and size of actuators and sensors (as well as on the type of controller used). The problem of optimal distribution of piezoelectric actuator patches has been studied by many authors (see [5] for a review), but since these approaches usually assume that size, shape, and number of actuators are given a priori, they may lead to sub-optimal solutions. It is also interesting to optimize both structural and control parameters at the same time. One of the first works in this area was ref. [2] which used fixed number and position of actuators for actively controlled beam structures.

Recently, some authors have begun to apply topology optimization to find optimal piezo-actuator distributions on plates and shells in the static case [14] and beams in the dynamic case [16], assuming constant thickness in all 
of them. In [15] some interesting results are obtained by using shape optimization techniques. In that work, an iterative technique to optimize the shape of piezoelectric actuators over beams and plates is used in order to achieve desired shapes of the structure.

Above discussed studies considered bulk piezoelectric materials. However, it is also possible to improve piezoelectric performance characteristics by designing an optimal periodic piezo-composite. Unit cell and homogenization based topology optimization studies can be found in [19] for the two-dimensional case as well as in [17] for the three-dimensional case.

In this paper, we consider an initially undamped cantilever beam with twin symmetrical surface bonded piezoelectric actuators uniformly distributed along the structure, a so-called piezoelectric bimorph actuator which is shown in Fig. 1. Firstly, we tackle the design problem of optimal distribution of piezo-actuator thickness along the structure subjected to time-harmonic loading in its free extreme, so that the tip-deflection can be controlled. In such a case, the design variable will be the piezo-actuator thickness, $h_{a}(x)$, (see Fig. 1a). The second design problem deals with finding the optimal distribution of piezo-actuator width along the structure under the same loading conditions, but now keeping constant the thickness. In Fig. 1b both side and top views of the composite structure are shown, with the piezo-actuator width, $w_{a}(x)$, being the new design variable. Starting with a uniform design variable distribution (constant width or thickness) the optimization results in the optimal piezo-actuator distribution including number of patches and their optimal placement. It is important to point out that both piezoelectric actuators in Fig. 1 have been poled in the same direction and hence, polarity will not be considered as a design variable in the design problem.

In relation to the thickness study, we can find a recent application in [3], where a variable thickness piezoelectric bimorph actuator is proposed for application to minimally invasive surgery (five discrete thicknesses are considered rather than continuous variation).

The paper is organized as follows: first we briefly present the governing equations for piezoelectric materials, compute the bending moment produced by the piezoactuators depending on the design variable, and emphasize the problems associated with constant driving voltage. In the latter case, a model connecting both field and thickness is required and therefore proposed here. Then, we obtain the optimal values for both design variables, thickness and width, that make the static tip-deflection zero. Later on, we formulate the optimal design problem in dynamics from the topology optimization perspective and finally, we show several numerical examples for a single driving frequency as well as for frequency intervals by using Padé approximants.

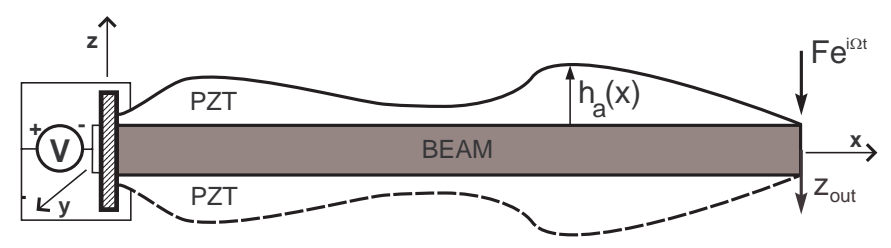

(a) Variable piezo-actuator thickness
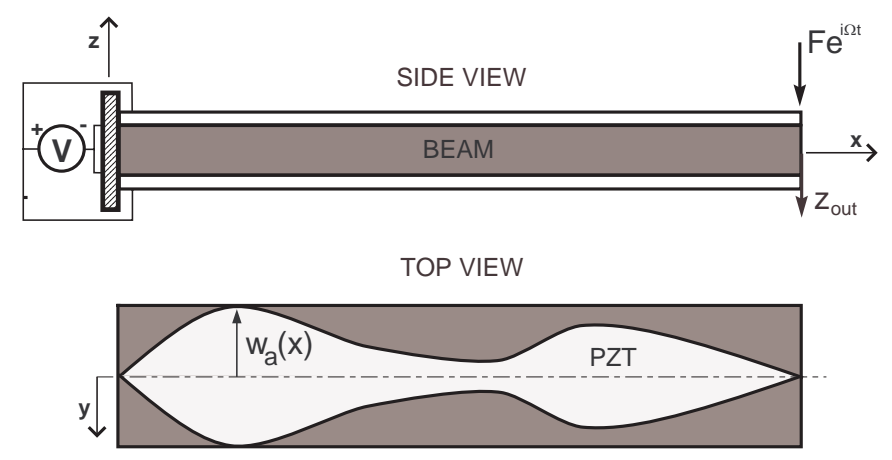

(b) Variable piezo-actuator width

Fig. 1 Design domain for both design problems

\section{Constitutive equations of piezoelectric materials}

We consider linear piezoelectricity, that is, piezoelectric materials which have a linear response under changes in the electric field, electric displacement, mechanical stress and strain. The relationships among all these tensors can be fully described by a single pair of electromechanical equations. There are many equivalent ways to write them and the best choice will depend on the problem studied. Using the notation of $[6]^{1}$, we can describe the behavior of a piezoelectric material by the following piezoelectric constitutive equations

$\left\{\begin{array}{l}\varepsilon_{i j}=s_{i j k l}^{E} \sigma_{k l}+d_{k i j} E_{k} \\ D_{i}=d_{i k l} \sigma_{k l}+\epsilon_{i k}^{\sigma} E_{k},\end{array}\right.$

where $i, j, k, l$ take on values $1,2,3$ (or $\mathrm{x}, \mathrm{y}, \mathrm{z}$ ), and $\varepsilon_{i j}$ is the strain tensor, $\sigma_{k l}$ is the stress tensor, $s_{i j k l}^{E}$ is the compliance tensor, $d_{k i j}$ are piezoelectric constants, $E_{k}$ is the electric field (also called electric strength in this context), $D_{i}$ is the electric displacement, and $\epsilon_{i k}^{T}$ is the permittivity. The superscripts $E$ and $\sigma$ indicate that the values of the tensors are obtained at constant electric field and constant stress, respectively.

In the absence of external loading and assuming that the electrostatic field is applied out of phase to the two piezo-actuators in the z-direction only (otherwise, the whole structure would be working in tension or compression), the beam undergoes pure bending around the

${ }^{1}$ We have replaced $S$ by $\varepsilon$, and $T$ by $\sigma$ for the sake of clarity. 

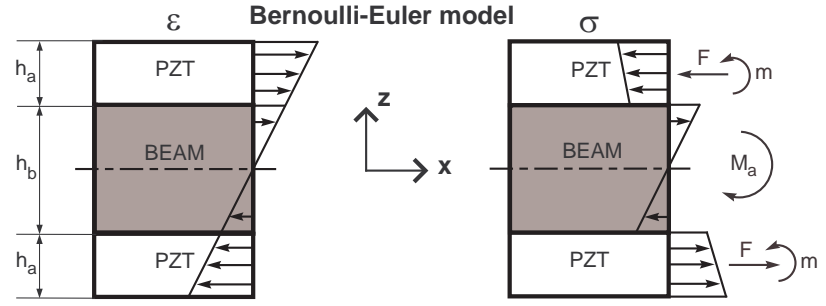

Fig. 2 Strain and stress distributions in a cross-section of the composite beam structure

y-axis (see Fig. 1). Now considering perfect bonding between beam and PZTs as well as Bernoulli-Euler beam theory [4], the composite structure undergoes linear strain, as it is shown in Fig. 2. This strain distribution assumes upward poling direction for both piezo-actuators as well as the polarity of the voltage source shown in Fig. 1. According to this, it is noticed that the piezo-actuator of the top becomes longer in the x-direction as well as thinner in the z-direction (while the piezo-actuator of the bottom becomes shorter in the x-direction and thicker in the $\mathrm{z}$-direction), however, the thickness change can be neglected since the piezo-actuator thickness value is much lower than its length. Consequently, the stresses will be confined in the x-direction, making the piezo-actuator of the top work in both compression and bending, while the one of the bottom work in tension and bending. Using a compact notation [6] it is not difficult to see that for this particular situation (1) reduces to the two following scalar equations

$\left\{\begin{array}{l}\varepsilon_{1}=\left(Y_{a}\right)^{-1} \sigma_{1}+d_{31} E_{3} \\ D_{3}=d_{31} \sigma_{1}+\epsilon_{33}^{\sigma} E_{3},\end{array}\right.$

where $Y_{a}$ is the Young's modulus of the piezoelectric material.

It is worthwhile emphasizing that the double-sided configuration of piezoelectric actuators fixes the neutral axis to the center of the beam.

2.1 Bending moment under constant electric field strength, $E_{3}$

Let us now consider both piezoelectric actuators of dimensions $L \times w_{a} \times h_{a}$, length, width and thickness, respectively (all of them constant values), surface bonded to a beam of dimensions $L \times w_{b} \times h_{b}$. Whenever an electrostatic field, $E_{3}=\frac{V_{a}}{h_{a}}$, is applied out of phase to both piezoelectric actuators, the deformation in the piezoelectric materials produces a pure bending moment $M_{a}$ acting on the beam (see Fig. 2), equal to the one produced

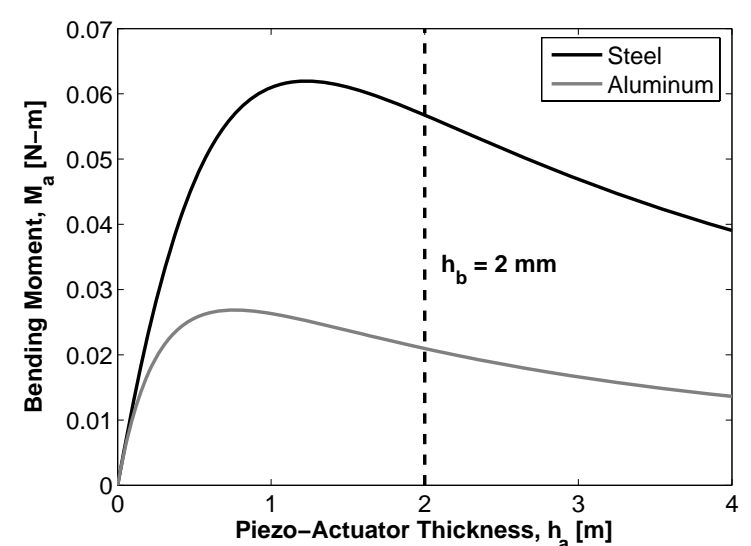

(a)

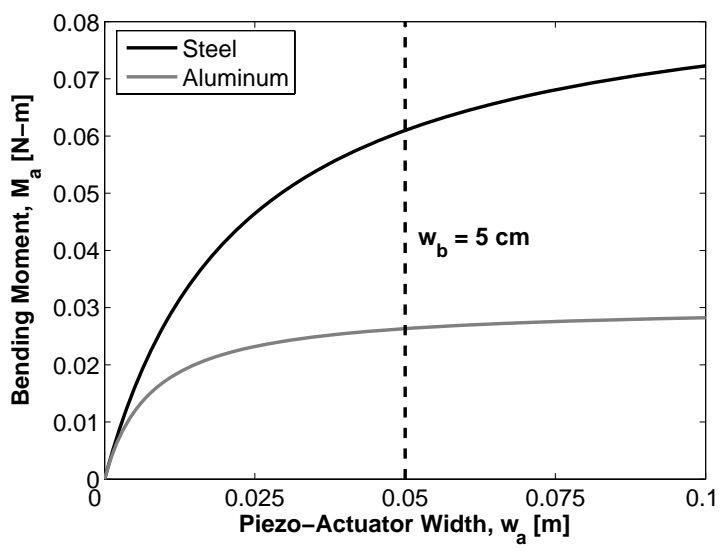

(b)

Fig. 3 Bending moment under constant electric field strength, $E_{3}$

by the beam in order to be in equilibrium, and easily computed by the formula

$$
\begin{aligned}
& M_{a}=2 \int_{\frac{h_{b}}{2}}^{\frac{h_{b}}{2}+h_{a}} \sigma_{1}(z) w_{a} z d z= \\
& \frac{w_{a} w_{b} Y_{a} h_{a}\left(h_{a}+h_{b}\right) Y_{b} h_{b}^{3} d_{31} E_{3}}{w_{a}\left(8 Y_{a} h_{a}^{3}+12 Y_{a} h_{b} h_{a}^{2}+6 Y_{a} h_{b}^{2} h_{a}\right)+w_{b} Y_{b} h_{b}^{3}} .
\end{aligned}
$$

In Fig. 3a and Fig. 3b bending moment functions are plotted versus the piezo-actuator thickness (under constant $w_{a}$ ) and piezo-actuator width (under constant $\left.h_{a}\right)$, respectively, for two beams of unit length $(L=1$ $\mathrm{m})$, thickness $h_{b}=2 \mathrm{~mm}$, width $w_{b}=5 \mathrm{~cm}$, and different Young's modulus (steel, $Y_{b}=200 \mathrm{GPa}$, and aluminum, $Y_{b}=70 \mathrm{GPa}$ ). Material properties of PZT are $Y_{a}=65$ $\mathrm{GPa}, d_{31}=200 \cdot 10^{-12} \mathrm{~m} / \mathrm{V}$, and $E_{3}=100 \mathrm{~V} / \mathrm{mm}$. In the thickness study $w_{a}=5 \mathrm{~cm}\left(=w_{b}\right)$, whereas in the width study $h_{a}=1 \mathrm{~mm}$.

Looking at Fig. 3a, we can see that there exists an optimal thickness value $([12,13])$ that maximizes the bending moment (almost half the thickness of the beam assuming a steel beam and a quarter thickness for an aluminum beam, since the latter material is less stiff than 

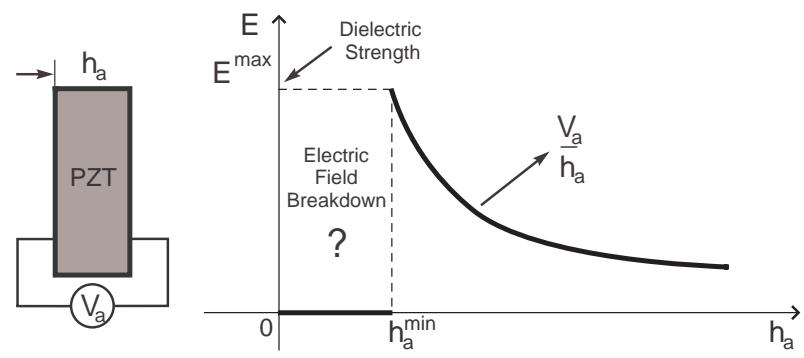

Fig. 4 PZT physics

steel). It is also observed how the bending moment function approaches zero when the thickness becomes large because the flexural rigidity of the piezo-actuator limits the bending strain. As opposed to that, Fig. 3b shows that the wider the piezo-actuator is, the higher the moment is as well, though it tends to a finite value (the less stiff the beam material, the lower such a value is) when the piezo-actuator width value becomes large.

\subsection{Bending moment under constant voltage, $V_{a}$}

We are now interested in understanding how the bending moment is affected when the amplitude voltage (again applied out of phase to both piezoelectric actuators) is constant rather than the amplitude field. For the moment, we will just focus on the thickness study.

It is known that whenever a voltage, $V_{a}$, is applied over the piezo-material electrodes of gap $h_{a}$ (see Fig. 4), electric energy is stored between them through the electric field produced, $E_{3}=\frac{V_{a}}{h_{a}}$. On decreasing the gap under the same voltage, the field increases its value. In practice, piezoelectric materials have a maximum allowable electric field strength $E^{\max }$ (also called dielectric strength) which, if exceeded, will degrade the material and eventually cause loss of piezoelectric properties. This is called electric field breakdown ([21]).

This discontinuity will cause problems for optimization, therefore we need to develop a model that connects both the electrostatic field, $E_{3}$, and the piezo-actuator thickness, $h_{a}$, in a smooth way; in other words, we are interested in finding a differentiable approximation between these two variables for any gap value. To do that, we propose the following interpolation functions

$E_{3}\left(h_{a}\right)=\frac{V_{a} h_{a}^{n}}{\left(h_{a}^{\min }\right)^{n+1}+h_{a}^{n+1}}$,

where $n$ is a given integer power such as $n \geq 1$, and $h_{a}^{\min }$ is a threshold value under which the piezoelectric material breaks down. The value of $h_{a}^{\mathrm{min}}$ is found by imposing that the thickness $h_{a}^{\star}$ verifying $E_{3}^{\prime}\left(h_{a}^{\star}\right)=0$, makes $E_{3}\left(h_{a}^{\star}\right)=E^{\max }$ hold. In that way, we arrive at

$h_{a}^{\min }=\frac{\left|V_{a}\right|}{\alpha(n) E^{\max }}$,

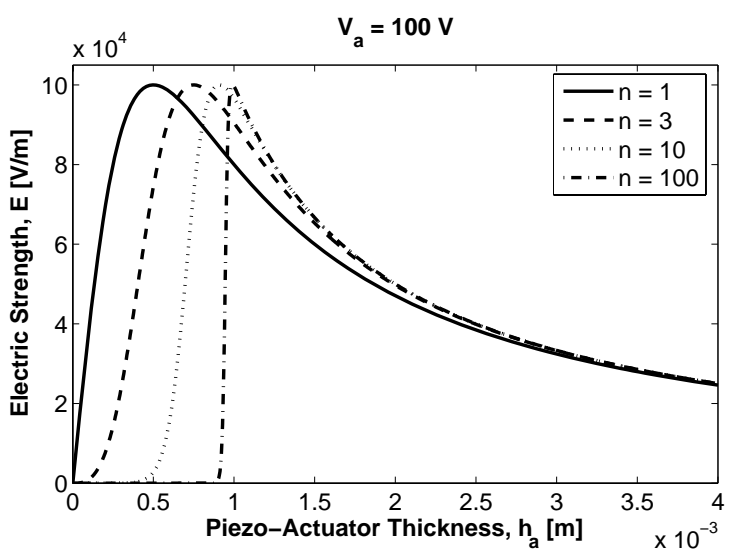

(a)

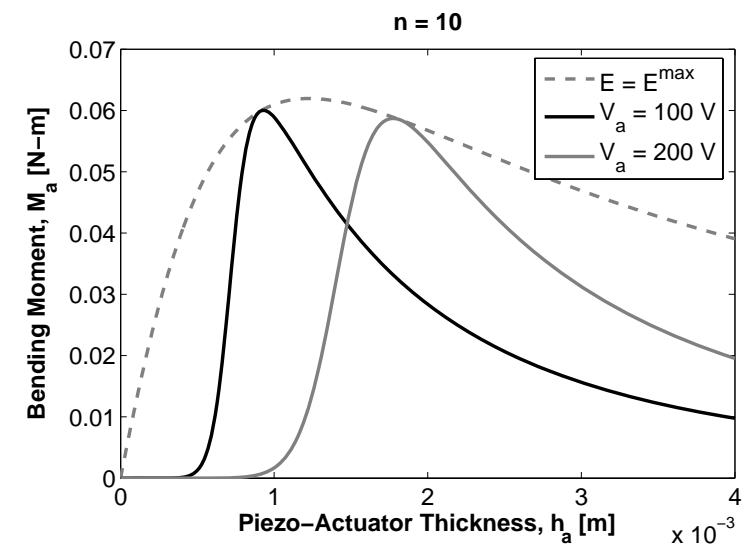

(b)

Fig. 5 Continuous piezoelectric response under constant voltage, $V_{a}$

where $\alpha(n)=\left(\frac{n+1}{\sqrt[n+1]{n^{n}}}\right)$. We can see in Fig. 5a how (4), on the one hand, takes the zero value when there is no piezo-actuator (and it also takes values close to zero when there is almost no piezo-actuator), and on the other hand, it tends to the commonly used expression $\frac{V_{a}}{h_{a}}$ when $h_{a} \gg h_{a}^{\mathrm{min}}$. For higher $n$, the smooth function approaches the original response in Fig. 4. As a consequence, the bending moment under constant voltage (see Fig. 5b) becomes a continuous and differentiable function and consistent with mechanical intuition

$$
\begin{aligned}
& M_{a}\left(h_{a}\right)=\frac{w_{a} w_{b} Y_{a} h_{a}\left(h_{a}+h_{b}\right) Y_{b} h_{b}^{3}}{w_{a}\left(8 Y_{a} h_{a}^{3}+12 Y_{a} h_{a}^{2} h_{b}+6 Y_{a} h_{a} h_{b}^{2}\right)+w_{b} Y_{b} h_{b}^{3}} \\
& \times d_{31} \frac{V_{a} h_{a}^{n}}{\left(h_{a}^{\min }\right)^{n+1}+h_{a}^{n+1}} .
\end{aligned}
$$

Bending moment functions, under constant electric field and under two constant values of voltage (for $n=10$ ), are also plotted in Fig. 5b, exhibiting the differences between both models. It is also important to notice that the maximum bending moment under constant voltage corresponds to the one obtained for the dielectric strength at that particular voltage value. 
When the design variable is the piezo-actuator width, $w_{a}$ the electric field is constant and does not depend on the design. For this case the bending moment under constant voltage is found by replacing the field term $E_{3}$ by the constant one $\frac{V_{a}}{h_{a}}$ in (3).

\section{Optimal solutions for the electrostatic case}

We reconsider the tip-loaded cantilever beam in Fig. 1, where both piezo-actuators have been poled now in the downward direction. From the previous section we can extract that either a homogeneous piezo-actuator thickness distribution or homogeneous piezo-actuator width distribution is equivalent to considering two couples of the same magnitude, one clockwise in the clamped extreme and another one counter-clockwise in the free extreme of the beam. Thus, we are interested in finding the optimal value of $t_{a}$ such that the bending moment $M_{a}$ is able to control the structure and hence, to get zero tip-displacement dependent on the tip-force $F$, that is,

$z_{\text {out }}\left(t_{a}\right)=\frac{L^{2}\left(3 M_{a}\left(t_{a}\right)-2 F L\right)}{6\left(Y_{b} I_{b}+2 Y_{a} I_{a}\left(t_{a}\right)\right)}=0$,

where $t_{a}$ is either homogeneous thickness $h_{a}$ or homogeneous width $w_{a}$. Notice that, in both cases, the design variable appears in the bending moment, as well as in the moment of inertia term. However, the design variable $t_{a}$ only needs to satisfy the equation $M_{a}\left(t_{a}\right)=\frac{2}{3} F L$.

Tip-deflection curves versus piezo-actuator thickness are plotted in Fig. 6a for different load values, $F$, under constant electric field. Depending on the loading, it is possible to control the output (make the tip-displacement zero). However, for $F>0.08 \mathrm{~N}$ it is only possible in the limit where the actuator thickness approaches infinity (i.e. the beam has infinite stiffness). In Fig. $6 \mathrm{~b}$ the same family of curves are plotted but now for $F=0.04 \mathrm{~N}$ and using different voltage values, $V_{a}$. As before, it is observed that sometimes $\left(V_{a}<25 \mathrm{~V}\right)$ it is not possible to get zero tip-displacement for finite thickness values. Finally, in Fig. 6c tip-deflection curves versus piezoactuator width are also shown for different load values and, as before, for $F>0.08 \mathrm{~N}$, is not possible for finite width values.

\section{Design problem formulation for harmonic loading}

Assuming very slender beams and lower order modes, we can use the classical Bernoulli-Euler's theory in the model for beam vibrations

$$
\frac{\partial^{2}}{\partial x^{2}}\left(Y I \frac{\partial^{2} \mathcal{W}}{\partial x^{2}}\right)+\rho A \frac{\partial^{2} \mathcal{W}}{\partial t^{2}}=F e^{i \Omega t} \delta(x-L)-\frac{\partial^{2} M_{a}}{\partial x^{2}}
$$

where we assume harmonic loading with driving frequency $\Omega$. The design variable, $t_{a}$ (either $h_{a}$ or $w_{a}$ ), is included

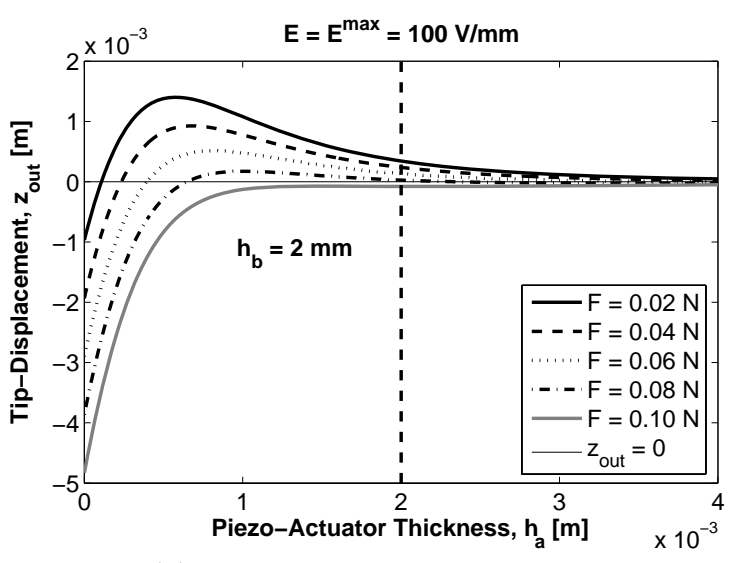

(a) Constant electrostatic field

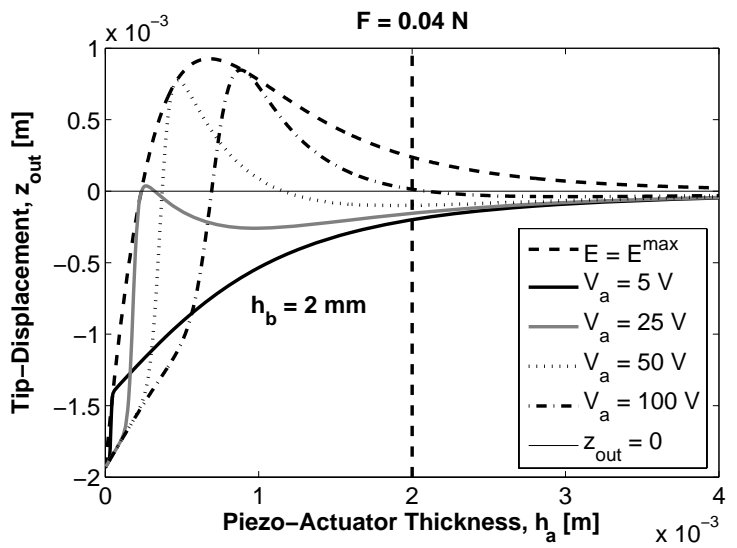

(b) Constant voltage

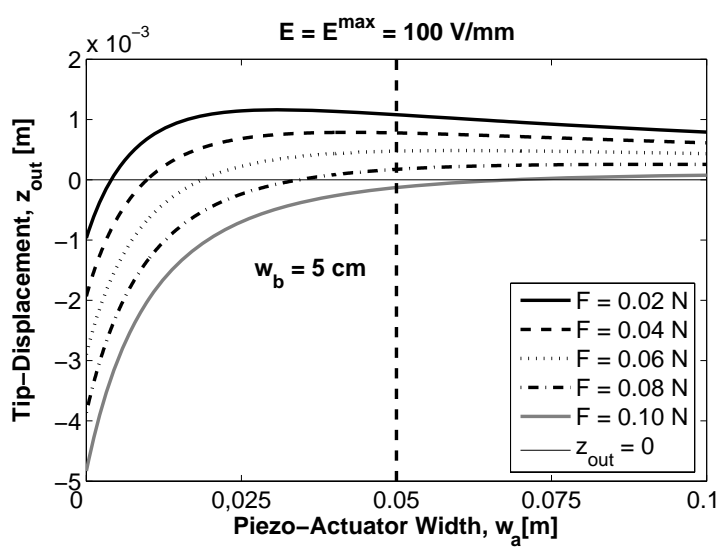

(c) Constant electrostatic field

Fig. 6 Optimal solutions for the electrostatic case

in the total stiffness, $Y I=2 Y_{a} I_{a}\left(t_{a}\right)+Y_{b} I_{b}$, in the total mass per unit length, $\rho A=2 \rho_{a} A_{a}\left(t_{a}\right)+\rho_{b} A_{b}$, as well as in the bending moment produced by the distributed piezo-actuator, $M_{a}\left(t_{a}\right)$.

Under harmonic loading (and either harmonically varying electric field or voltage as well), the vertical displacement will also be harmonic,

$\mathcal{W}(x, t)=z(x) e^{i \Omega t}$ 
where $z(x)$ is the amplitude function, verifying the ordinary differential equation

$\left(Y I z^{\prime \prime}(x)\right)^{\prime \prime}-\Omega^{2} \rho A z(x)=F \delta(x-L)-M^{\prime \prime}(x)$,

and $M$ is the bending moment in which the harmonic term concerning either the electric field or voltage has been canceled when we take derivatives with respect to the $x$-coordinate.

Under all these considerations and adding a volume constraint on the piezoelectric material as well as bound constraints on the design variables, the design problem is mathematically formulated as

$\min _{t_{a}}:\left(z_{\text {out }}\right)^{2}$,

subject to

$$
\begin{aligned}
\left(Y I\left(t_{a}\right) z^{\prime \prime}(x)\right)^{\prime \prime}-\Omega^{2} \rho A\left(t_{a}\right) z(x) & =-M^{\prime \prime}\left(t_{a}\right), \text { in }(0, L) \\
z(0)=z^{\prime}(0) & =0 \\
-\left(Y I\left(t_{a}\right) z^{\prime \prime}(x)\right)^{\prime}(L) & =F \\
-\left(Y I\left(t_{a}\right) z^{\prime \prime}(x)\right)(L) & =0 \\
\frac{1}{L} \int_{0}^{L} t_{a} d x & \leq f_{a} t_{a}^{\max } \\
0 \leq t_{a} & \leq t_{a}^{\max }
\end{aligned}
$$

where $t_{a}^{\max }$ and $f_{a}\left(0<f_{a}<1\right)$ are the given upper bound and upper volume fraction for the piezoelectric material, respectively.

Even though we are considering a shape optimization problem rather than a genuine topology optimization one (the reader is referred to [1] for an overview of the method and different applications), the philosophy is basically the same. The method used is a gradient-based optimization algorithm $([20])$ that solves the problem of distributing a limited amount of material in a design domain in order to optimize a certain objective function. The standard approach consists of discretizing the design domain in finite elements and letting each one of them have a variable density $\rho^{e}$ as design variable. Any variable can vary from zero to one, and typically represents a spatial material density.

For our problem, having discretized the design domain in $N$ finite elements, the densities appear when we normalize the design variables in the following way

$t_{a}^{e}=t_{a}^{\max } \rho_{a}^{e}, 0 \leq \rho_{a}^{e} \leq 1, e=1, \cdots, N$.

After discretization, the optimization problem can be written as

$\min _{\boldsymbol{\rho}}: \mathbf{U}^{T} \mathbf{L} \mathbf{U}$

subject to

$$
\begin{aligned}
{\left[\mathbf{K}(\boldsymbol{\rho})-\Omega^{2} \mathbf{M}(\boldsymbol{\rho})\right] \mathbf{U} } & =\mathbf{F}(\boldsymbol{\rho}), \\
\mathbf{v}^{T} \boldsymbol{\rho} & \leq N f_{a} \\
\mathbf{0} & \leq \boldsymbol{\rho} \leq \mathbf{1}
\end{aligned}
$$

where $\mathbf{L}$ is a zero matrix with ones at the diagonal elements corresponding to the output nodes, $\mathbf{U}$ and $\mathbf{F}$ are the global displacements and force vectors, respectively, $\mathbf{K}$ and $\mathbf{M}$ are the global stiffness and mass matrices, respectively, $\boldsymbol{\rho}$ is the vector of design variables, and $\mathbf{v}$ is a vector containing the volume of the elements. This formulation was first adopted in [18].

To perform the sensitivity analysis, we first rewrite the objective function by adding a zero term (according to the adjoint method)

$c(\boldsymbol{\rho})=\mathbf{U}^{T} \mathbf{L} \mathbf{U}+\boldsymbol{\lambda}^{T}\left(\left[\mathbf{K}(\boldsymbol{\rho})-\Omega^{2} \mathbf{M}(\boldsymbol{\rho})\right] \mathbf{U}-\mathbf{F}(\boldsymbol{\rho})\right)$.

Taking into account that the displacement field is regarded as a function of the design variables through the equilibrium equation, we obtain the derivatives of the objective function with respect to the densities,

$\frac{\partial c}{\partial \boldsymbol{\rho}}=\boldsymbol{\lambda}^{T}\left(\frac{\partial \mathbf{K}}{\partial \boldsymbol{\rho}}-\Omega^{2} \frac{\partial \mathbf{M}}{\partial \boldsymbol{\rho}}\right) \mathbf{U}-\boldsymbol{\lambda}^{T} \frac{\partial \mathbf{F}}{\partial \boldsymbol{\rho}}$,

with $\boldsymbol{\lambda}$ satisfying the adjoint equation

$\left[\mathbf{K}(\boldsymbol{\rho})-\Omega^{2} \mathbf{M}(\boldsymbol{\rho})\right] \boldsymbol{\lambda}=-2 \mathbf{L} \mathbf{U}$.

\section{Numerical examples}

In this section, we illustrate our approach through several numerical examples for a steel beam. We begin by finding optimized distributions of variable thickness under constant electric field amplitude, $E_{3}=100 \mathrm{~V} / \mathrm{mm}$.

In Fig. 7a an optimized thickness profile is shown for $F=0.04 \mathrm{~N}$ and $f=(\Omega / 2 \pi)=1.9 \mathrm{~Hz}$, when $h_{a}^{\max }=$ $h_{b}=2 \mathrm{~mm}$, and the volume constraint is $f_{a}=0.5$. The dashed black line corresponds to the beam and the grey one is the optimized solution obtained through the numerical algorithm. In this particular case, the obtained solution consists of considering a piezoelectric material distribution whose thickness varies as it is shown in Fig. 7a. At this point, it is important to notice that it not possible to make a piezoelectric material with variable thickness (like wedge shape, for instance) for a single layer type ceramic. However, using multilayer technology it becomes possible by subdividing the piezoelectric material in thin layers poled individually. In other words: once optimized designs have been obtained, a post-processing step would be required to make the manufacturing process possible (see the final design proposed in Fig. 7a). Another possibility is to discretize the optimized solution in finite thicknesses. That is precisely indicated with the black line in Fig. 7b, where two piezo-actuator patches of different thickness value have been chosen to construct the final design for $f=11.1 \mathrm{~Hz}$ (below second mode). Another example is shown in Fig. 7c by using the same input data but driving frequency $f=29.4 \mathrm{~Hz}$ (below third mode). This time more discrete thicknesses are needed (only four are considered) to reflect as much as possible the optimized solution. Judging from the numerical results, it also seems that we would need more patches as the driving frequency increases its value, but, of course, 


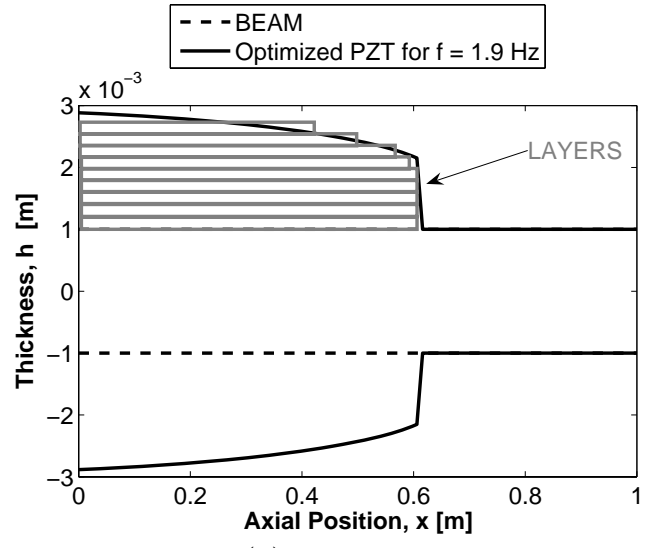

(a) $f=1.9 \mathrm{~Hz}$

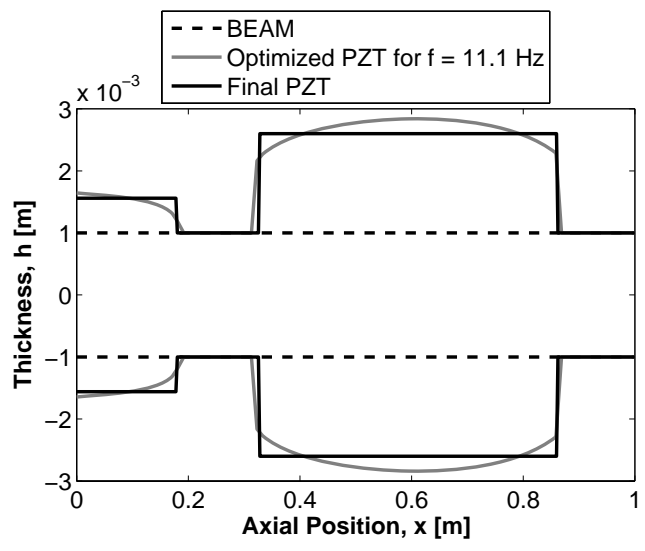

(b) $f=11.1 \mathrm{~Hz}$

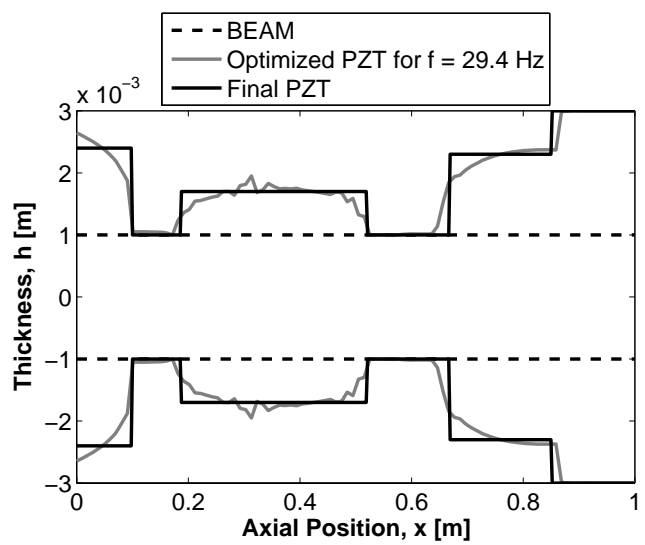

(c) $f=29.4 \mathrm{~Hz}$

Fig. 7 Optimized solutions under constant electric field amplitude

the objective function should be compared after modifications.

Anyway, common to all three situations is the fact that, keeping constant the electric field amplitude, since $E_{3}=\frac{V_{a}}{h_{a}}$, under variable thickness $h_{a}$, the voltage has also to vary along the structure, so it means that to control the structure, we would need the same number of voltage sources as the layers or discrete piezo-actuators used. More specifically, in the two previous examples, at least, two and four voltage sources would be necessary, respectively. Again, on increasing the driving frequency, more voltage sources would be expected, which does not seem to be a viable way to proceed, from an engineering point of view.

The manufacturing problem can be overcome by keeping the driving voltage rather than the driving field constant. The last two examples are now shown with the frequency response in Fig. 8 under constant amplitude voltage, $V_{a}=50 \mathrm{~V}$. In Fig. $8 \mathrm{~b}$ it can be observed how there are slight differences between frequency response of both optimized and final design (after using the second post-process mentioned before) around the driving frequency, $f=11.1 \mathrm{~Hz}$. However, more changes than before are noted in Fig. 8d, even though both frequency responses take the same value for the new design frequency $f=29.4 \mathrm{~Hz}$. This clearly tells us how sensitive the frequency response is with respect to small variations of the optimized designs, and how exact the postprocessing should be, otherwise, we will run the risk of being far from our initial objective.

Considering the width case, optimized distributions of piezo-actuator width (under constant thickness, $h_{a}=$ $1 \mathrm{~mm}$ ) are shown in Fig. 9a and Fig. 9c for $F=0.04 \mathrm{~N}$, $w_{a}^{\max }=w_{b}=5 \mathrm{~cm}$, and $f=10.3 \mathrm{~Hz}$ and $f=29.4 \mathrm{~Hz}$, respectively, when the amount of piezo-actuator is limited to $25 \%\left(f_{a}=0.25\right)$. It can be observed (see Fig. $9 \mathrm{~b}$ and Fig. 9d) how the frequency response is optimized at those particular driving frequencies, but a small variation in the frequency response would imply an output far from the optimized one. This justifies why it will be more appropriate to minimize the frequency response for a range of frequencies rather than for a single frequency. An efficient way to do this is by using Padé approximants ([7], [8]), because they provide an accurate frequency response in a design interval at low computational cost. Basically, a Padé approximant is that rational function (of a specified order) whose power series agrees with a given power series (it is typically an unknown function) to the highest possible order. In our context, the unknown function is the vector which contains the discretized nodal values of the amplitude function, U. Truncating the Taylor expansion of such an amplitude function near the design frequency $\Omega_{0}$ and replacing it by its corresponding Padé approximant (the Taylor expansion has a very limited convergence range near $\Omega_{0}$, regardless of a large number of expansion coefficients), the frequency response is accurately approximated for a relatively small number of expansion terms. 


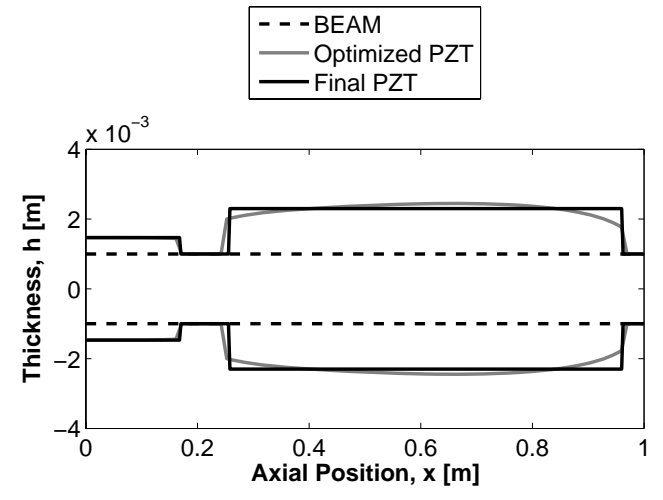

(a)

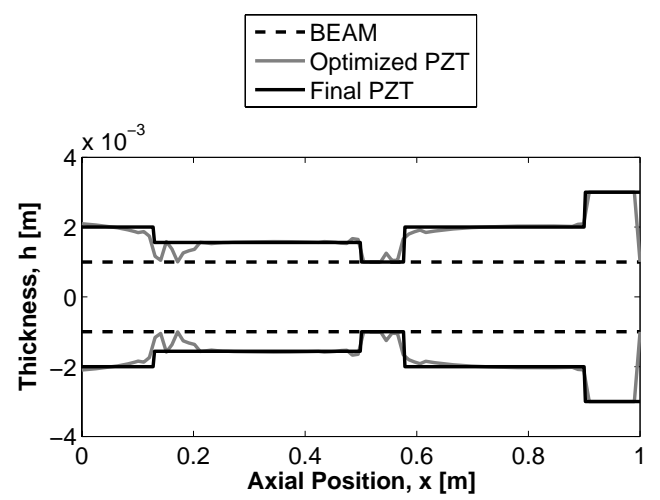

(c)

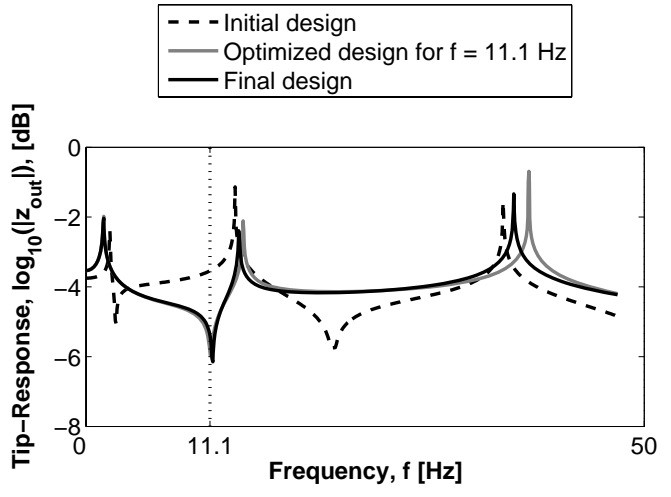

(b)

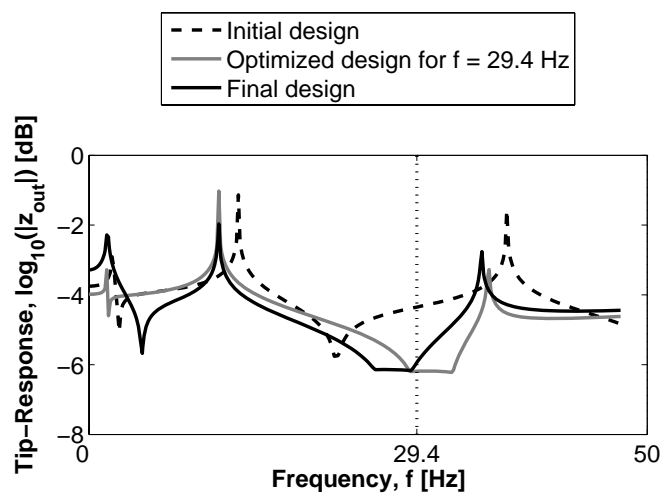

(d)

Fig. 8 Optimized solutions under constant voltage amplitude for $f=11.1 \mathrm{~Hz}$ (top) and $f=29.4 \mathrm{~Hz}$ (bottom).

The new objective function is the average response in the frequency range $\Omega \in\left[\Omega_{0}-\Delta \Omega, \Omega_{0}+\Delta \Omega\right]$, that is,

$$
\begin{aligned}
& c=\frac{1}{2 \Delta \Omega} \int_{\Omega_{0}-\Delta \Omega}^{\Omega_{0}+\Delta \Omega} \mathbf{U}^{T} \mathbf{L} \mathbf{U} d \Omega \approx \\
& \frac{1}{N_{\Omega}} \sum_{k=1}^{N_{\Omega}}\left(\mathbf{U}^{p}\right)^{T} \mathbf{L}\left(\mathbf{U}^{p}\right),
\end{aligned}
$$

where $\mathbf{U}^{p}$ is the Padé approximant of the Taylor expansion of $\mathbf{U}$ and $N_{\Omega}$ is the number of evaluation points in the design interval. Although the computation of the new sensitivity analysis is rather tedious, analytical expressions can be obtained. We will not go into details here about that, but the reader is referred to [8] and [9] for a complete analysis of the sensitivity as well as other aspects related to the numerical procedure.

In Fig. 10a and Fig. 10c optimized distributions for piezo-actuator width are shown for the frequency intervals $\left[\begin{array}{ll}10 & 13\end{array}\right] \mathrm{Hz}$ and $\left[\begin{array}{ll}6.8 & 16.4\end{array}\right] \mathrm{Hz}$, respectively. As it is shown in Fig. 10b and Fig. 10d, the frequency response of the output is now optimized in the whole initial interval (in black) and therefore, transformed into a new one (in grey).
In Fig. 11 optimized distributions that minimize the tip-response around the third mode in the intervals [29.4 35.8] $\mathrm{Hz}$ and [26 39] $\mathrm{Hz}$ are also shown.

Finally, we minimize the tip-response in the whole interval [0 48] Hz. In this case, three Padé approximants (centered on $7.9 \mathrm{~Hz}, 23.8 \mathrm{~Hz}$ and $39.8 \mathrm{~Hz}$, respectively) are needed to accurately approximate the frequency response in the design interval. An optimized profile is shown in Fig. 12a when the starting point is the usual homogeneous distribution of volume 0.3 and $f_{a}=0.3$, but the final volume of the solution is 0.21 (i.e. the volume constraint is not active). In Fig. $12 \mathrm{~b}$ we can see how the frequency response is minimized in all the interval. Now, if this optimized solution is used as a new starting point with $f_{a}=0.21$, then a new optimized solution of final volume 0.21 (now the volume constraint is active) that gives us a lower value of the objective function is obtained (see Fig. 12c and Fig. 12d). However, a worse solution (in terms of our objective function) is obtained by starting with a homogeneous distribution of volume 0.25 and $f_{a}=0.25$ (see Fig. 12e and Fig. 12f). We have also noticed in other examples that different solutions that gives us similar values of the objective function are 


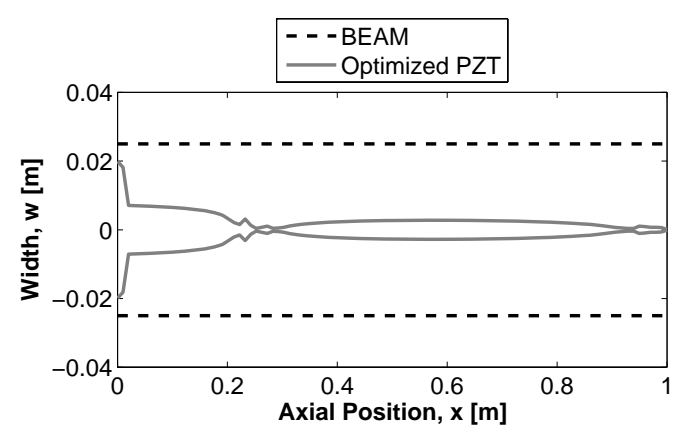

(a)

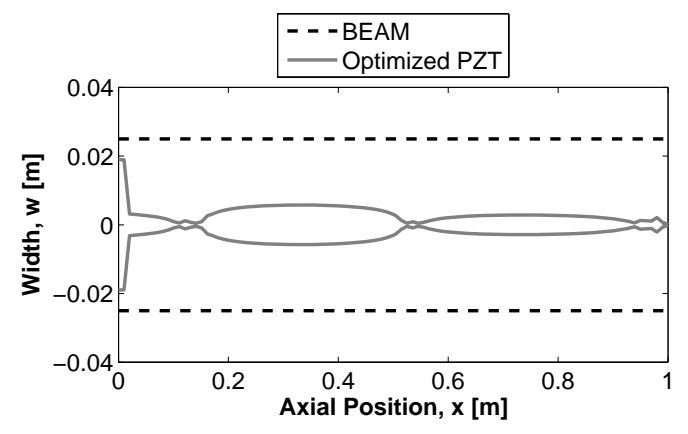

(c)

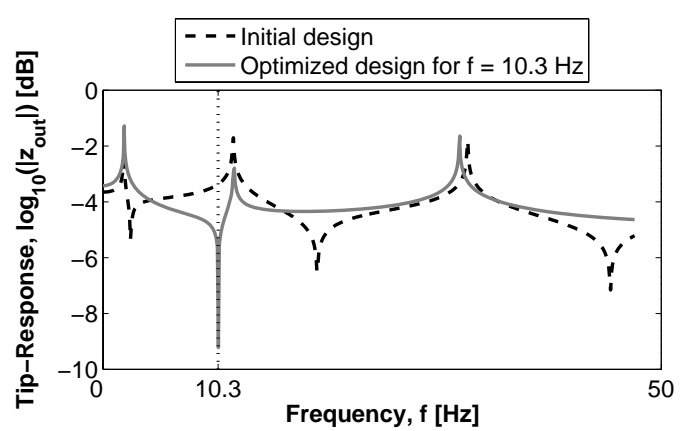

(b)

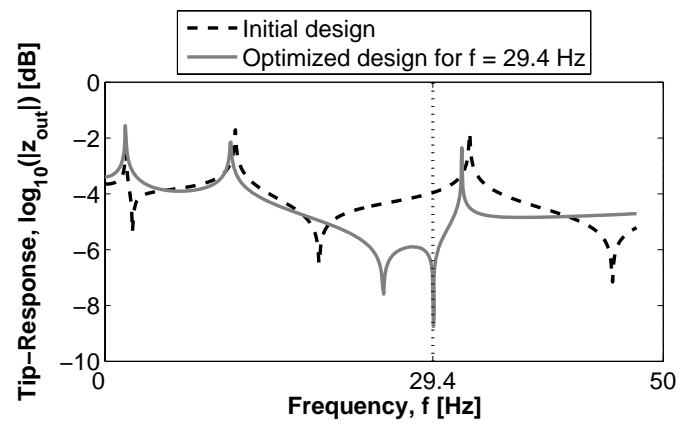

(d)

Fig. 9 Optimized solutions under constant voltage amplitude for $f=10.3 \mathrm{~Hz}$ (top) and $f=29.4 \mathrm{~Hz}$ (bottom).

obtained by changing the starting point. According to this, we can conclude that the design problem studied here is both non-unique and has many local minima.

This time (taking the width as design variable), the optimized shapes do not raise any manufactured difficulties if processed by e.g. laser cutting techniques, though sometimes a design regularization would be advisable to smooth resulting designs.

\section{Conclusions}

An optimal design problem in the context of active damping has been treated in this paper. The problem of controlling the tip-deflection of a cantilever beam subjected to time-harmonic loading on its free extreme has been studied, by independently optimizing two design parametrizations, namely thickness and width distribution of a piezoelectric bimorph actuator surface bonded to the structure. In the thickness study, the formulation depends on whether the electric field or the voltage is kept constant. In the latter case, a model connecting both electric field and piezo-actuator thickness is necessary. For manufacturing reasons we conclude that a post-processid step (either discretization in layers or in finite thicknesses) is required after finishing or during the optimization process. However, the constant voltage actuation is desirable because only one voltage source is needed to control the output of the beam. Another interesting observation is the fact that different thickness values can take the same bending moment value indicating nonuniqueness of the results.

For the width optimization case, non-uniqueness of designs remain a problem but manufacturing is not an issue. Therefore we find it more advisable to focus on the width as design variable rather than the thickness.

Finally, it is important to point out that optimization for a range of frequencies rather than for a single frequency value lets us obtain a really good broad band performance in the structure to be controlled, otherwise, the designs obtained are very sensitive to perturbations in design and loading frequency.

Acknowledgements This major part of this work was performed while AD was visiting the Department of Mechanical Engineering, DTU, Lyngby. The stay was supported by a post-doctoral fellowship of Junta de Comunidades de Castillaing Mancha (Spain). The work has also been supported by 


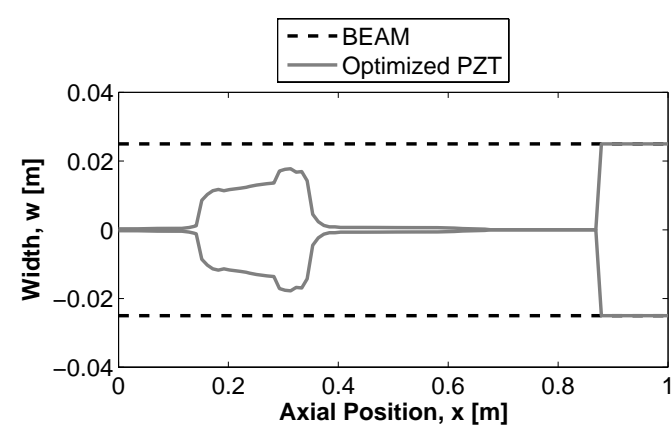

(a)

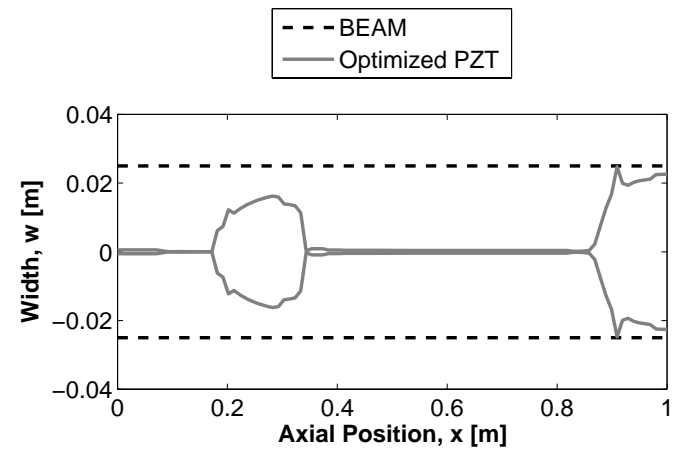

(c)

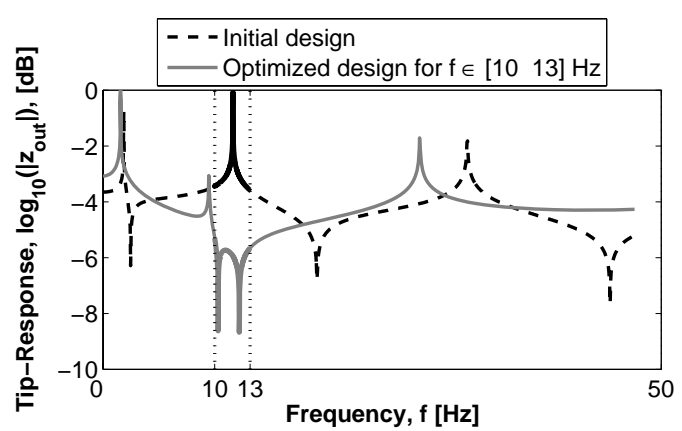

(b)

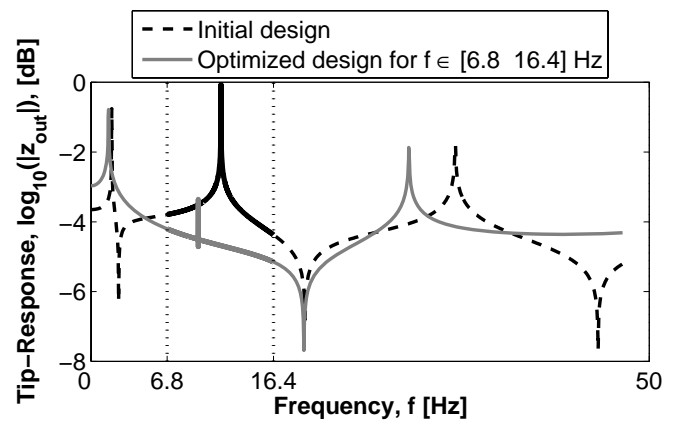

(d)

Fig. 10 Optimized solutions under constant voltage amplitude for $f \in\left[\begin{array}{lll}10 & 13\end{array}\right] \mathrm{Hz}$ (top) and $f \in[6.8$ 16.4 $] \mathrm{Hz}$ (bottom).

Ministerio de Educación y Ciencia MTM2004-07114 (Spain, AD), Junta de Comunidades de Castilla-La Mancha PAI-05027 (Spain, AD), Universidad de Castilla-La Mancha PCTC062 (Spain) and from an Eurohorcs/ESF European Young Investigator Award (EURYI, www.esf.org/euryi) through the grant "Synthesis and topology optimization of optomechanical systems" (EU, OS). The authors acknowledge stimulating discussions on the subject of this paper with Martin P. Bendsøe, Jacob S. Jensen, Gil-Ho Yoon and Jun S.O. Fonseca.

\section{References}

1. M.P. Bendsøe And O. Sigmund, Topology Optimization - Theory, Methods and Applications, Springer-Verlag, Berlin Heidelberg, 2003.

2. M.P. Bendsøe, N. Olhoff And J.E. Taylor, On the desing of structure and controls for optimal performance of actively controlled flexible structures, Mech. Struct. \& Mach., Vol. 15, N. 3, (1987), pp. 265-295.

3. D.J. Cappelleri, M.I. Frecker and T.W. Simpson, Design of a PZT Bimorph Actuator Using a MetamodelBased Approach, Journal of Mechanical Design, Vol. 124, (2002), pp. 354-357.

4. E. Crawley and E.H. Anderson, Detailed Models of Piezoceramic Actuation of Beams, Journal of Intelligent
Material System and Structures, Vol. 1 (1), (1990), pp. 415.

45. M.I. FreCKER, Recent Advances in Optimization of Smart Structures and Actuators, Journal of Intelligent Material Systems and Structures, Vol. 14, (2003), pp. 207-216.

6. IEEE Standard on Piezoelectricity, ANSI/IEEE Std. 1761987, 1988.

7. J.S. JENSEN AND O. Sigmund, Topology optimization of photonic crystal structures: a high bandwidth low-loss T-junction waveguide, Journal of the Optical Society of America B, Vol. 22, (2006), pp. 1191-1198.

8. J.S. JENSEN, Efficient optimization of dynamic systems using Padé approximants, III European Conference on Computational Mechanics Solids, Structures and Coupled Problems in Engineering, C.A. Mota Soares et. al (eds.) (2006), CD-rom.

9. J.S. JENSEN, Topology optimization of dynamic problems with Padé approximants, International Journal for Numerical Methods in Engineering (2007), in press.

10. J. Jaffe, R.S. Roth and S. MARZullo, Piezoelectric Properties of Lead Zirconate-Lead Titanate Solid-Solution Ceramics, Journal of Applied Physics, Vol. 25 , (1954), pp. 809-810.

11. H. KaWAI, The Piezoelectricity of Poly (vinylidene Fluoride), Japan. J. Appl. Phys., Vol. 8, (1969), pp. 975-976.

12. S.J. KIM AND J.D. JonES, Optimal design of Piezoactuators for Active Noise and Vibration Control, AIAA Journal, Vol. 29(12) (1991), pp. 2047-2053.

13. S.J. KIM AND J.D. JONES, Influence of Piezo-Actuator Thickness on the Active Vibration Control of a Cantilever 


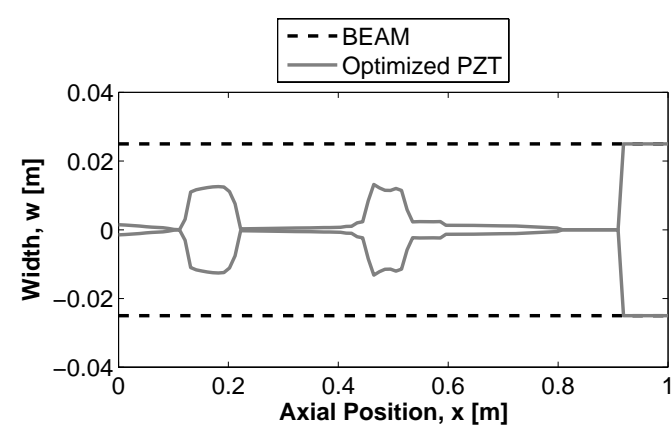

(a)

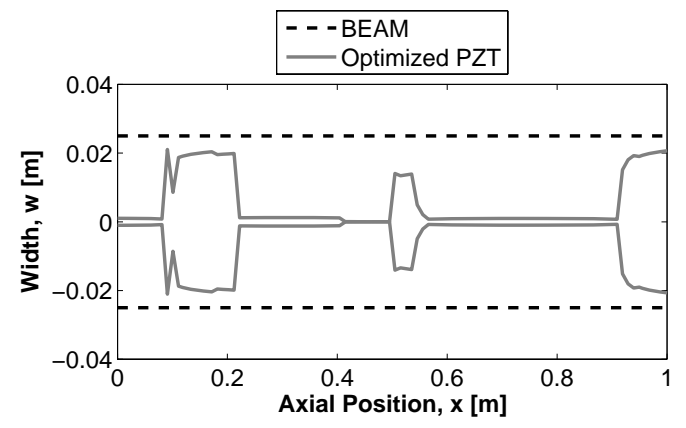

(c)

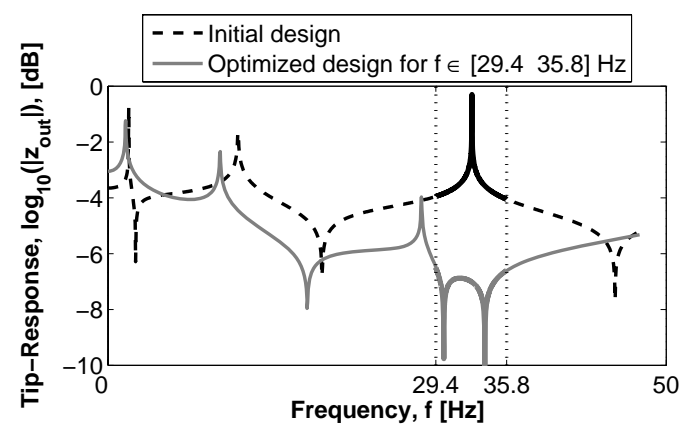

(b)

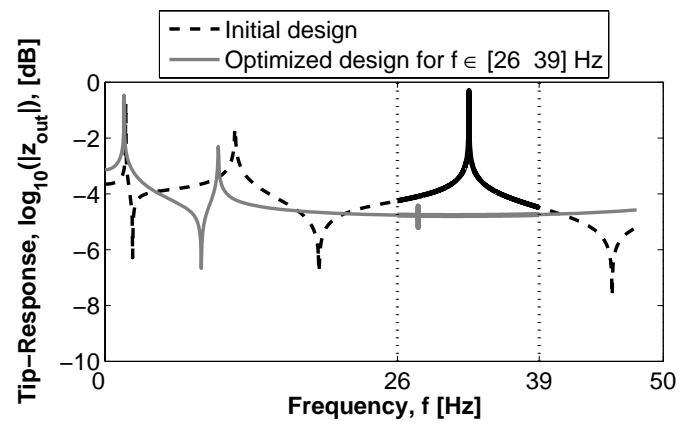

(d)

Fig. 11 Optimized solutions under constant voltage amplitude for $f \in[29.435 .8] \mathrm{Hz}$ (top) and $f \in[2639] \mathrm{Hz}$ (bottom).

Beam, Journal of Intelligent Material Systems and Structures, Vol. 6 (1995), pp. 610-623.

14. M. KÖGL AND E.C.N. SILVA, Topology optimization of smart structures: design of piezoelectric plate and shell actuators, Smart Mater. Struct., Vol. 14, (2005), pp. 387399.

15. A. MukherJee And S.P. Joshi, A gradientless technique for optimal distribution of piezoelectric material for structural control, Int. J. Numer. Meth. Engng., Vol. 57, (2003), pp. $1737-1753$.

16. M.J. Parsons, J. Jiang And A. Lumsdaine, Topology Optimization of active control vibration damping layers, Proceedings SPIE Smart Structures and Materials, Vol. 5757, (2005), pp. 153-163.

17. O. Sigmund, S. Torquato and I.A. Aksay, On the design of 1-3 piezocomposites using topology optimization, J. Mater. Res., Vol. 13, No. 4 (1998), pp. 1038-1048.

18. O. Sigmund AND J. Jensen, Systematic design of phononic band-gap materials and structures by topology optimization, Phil. Trans. R. Soc. Lond. A, Vol. 361, (2003), pp. 1001-1019.

19. E.C.N. Silva, J.S.O. Fonseca and N. Kikuchi, $O p$ timal design of piezoelectric microstrures, Computational Mechanics, Vol. 19, (1997), pp. 397-410.

20. K. SVANBERG, The method of moving asymptotes - a new method for structural optimization, Int. J. Numer. Meth. Eng., Vol. 24, (1987), pp. 359-373.

21. J.M. TORRES AND R.S. DharIWAL, Electric field breakdown at micrometre separations, Nanotechnology, Vol. 10, (1999), pp. 102-107. 


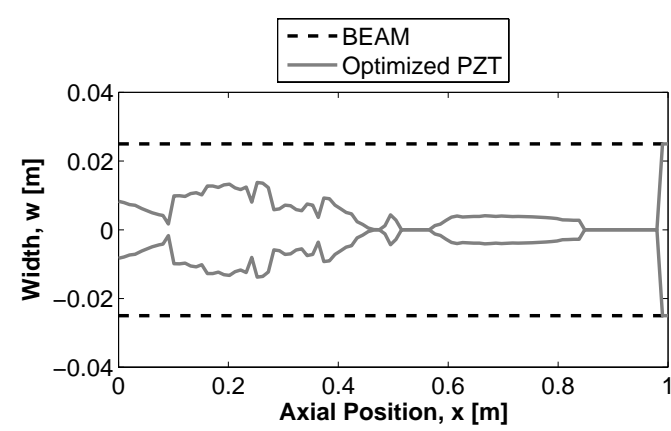

(a)

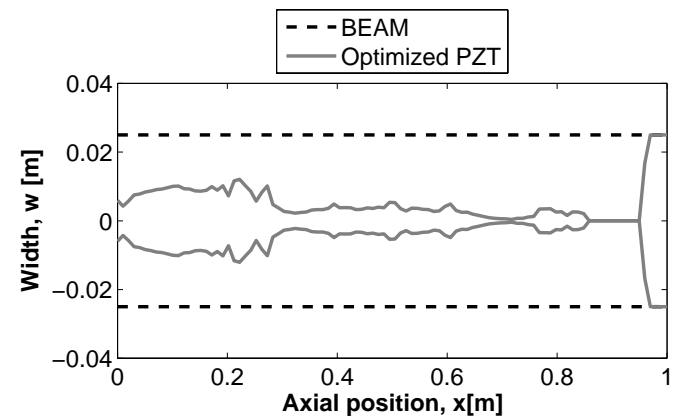

(c)

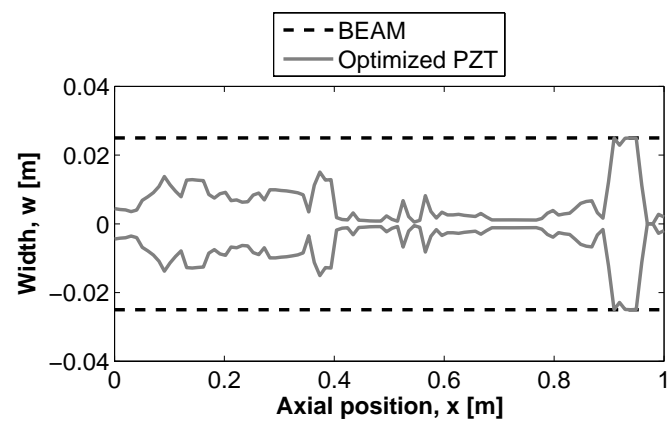

(e)

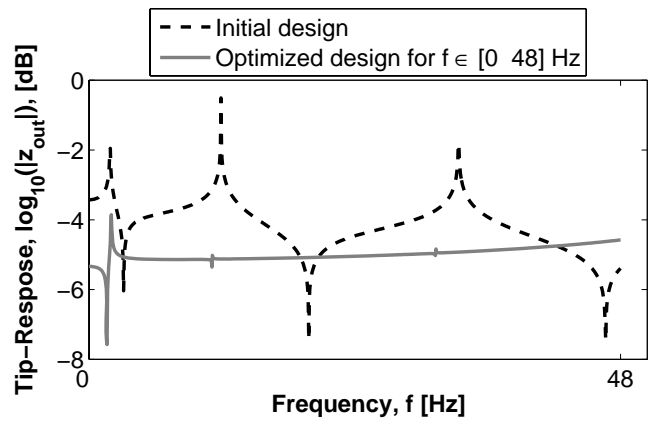

(b)

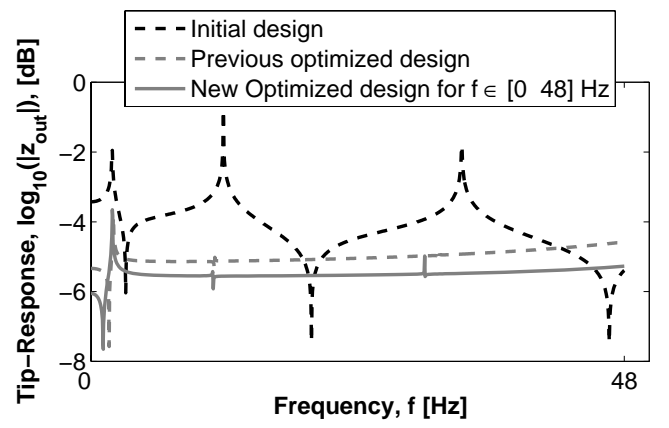

(d)

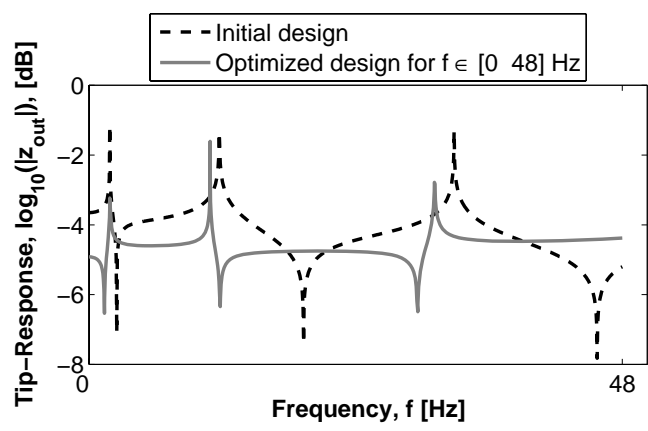

(f)

Fig. 12 Optimized solutions under constant voltage amplitude for $f \in[048] \mathrm{Hz}$, by using different upper volume fractions: $f_{a}=0.3$ (top), $f_{a}=0.20$ (middle), and $f_{a}=0.25$ (bottom). 